\title{
In Light of Visual Arts \\ A knowledge transfer partnership project as experiential learning
}

\section{Ming-hoi Lai}

Hong Kong Baptist University

Gateways: International Journal of Community Research and Engagement Vol 6 (2013): 218-27

(C) UTSePress and the author

ISSN 1836-3393
The 'In Light of Visual Arts' project was initiated by two academic units within the Hong Kong Baptist University (HKBU): the Academy of Visual Arts (AVA) and the Knowledge Transfer Office (KTO). These academic units partnered with MEGAMAN® Hong Kong, a light manufacturer, on this project. The three parties had a shared mission: to engage a wider audience for eco-friendly lights and to create awareness of their uses, so that the targeted audience, both visual arts students and the general public, would come to appreciate the importance of lighting effects to the visual arts sector and other creative industries. How the three parties were connected in this knowledge transfer partnership (KTP) project is examined below.

\section{PROJECT OBJECTIVES}

There were five main objectives of the 'In Light of Visual Arts' project: (1) to enhance students' ability in the use of lighting in visual arts communication; (2) to facilitate knowledge transfer of art appreciation and art archiving between manufacturers and art practitioners; (3) to raise and gain awareness of HKBU's contribution to visual communication and the environment; (4) to stimulate knowledge transfer in the arts and humanities through collaboration and partnerships; and (5) to make an impact by delivering the concept of 'eco-philosophy of light' through visual arts, research and documentation.

\section{THE PART NERSHIP}

According to the HKBU's Knowledge Transfer Office, knowledge transfer (KT) is 'the transfer of knowledge, expertise, skills and capabilities from universities as the academic knowledge base to companies or organizations in need of the knowledge, such as nongovernment organizations, commercial and industrial sectors, and various non-academic beneficiaries' (Knowledge Transfer Office 2013). KTO considers that KT should not be limited to technology transfer or commercialisation, but should include transfer of knowledge and know-how between academic disciplines such as Arts, Education and Humanities. 
Figure 1: Overview of the three parties' collaboration
To echo KTO advocacy, AVA and KTO, the two academic units within $\mathrm{HKBU}$, worked with MEGAMAN® Hong Kong to develop the 'eco-philosophy of light', a pilot scheme emphasising a participant-and-audience-oriented experience. Visual Arts Axis, the community art branch of AVA, was responsible for the project's execution, which aimed at reaching a general audience, including the communities near the two campuses of AVA in East Kowloon, as well as its local secondary school network. The three parties' involvement was as follows: Academy of Visual Arts of HKBU (academic partner); MEGAMAN® Hong Kong (external partner); Knowledge Transfer Office of HKBU (KT associate) - see Figure 1.

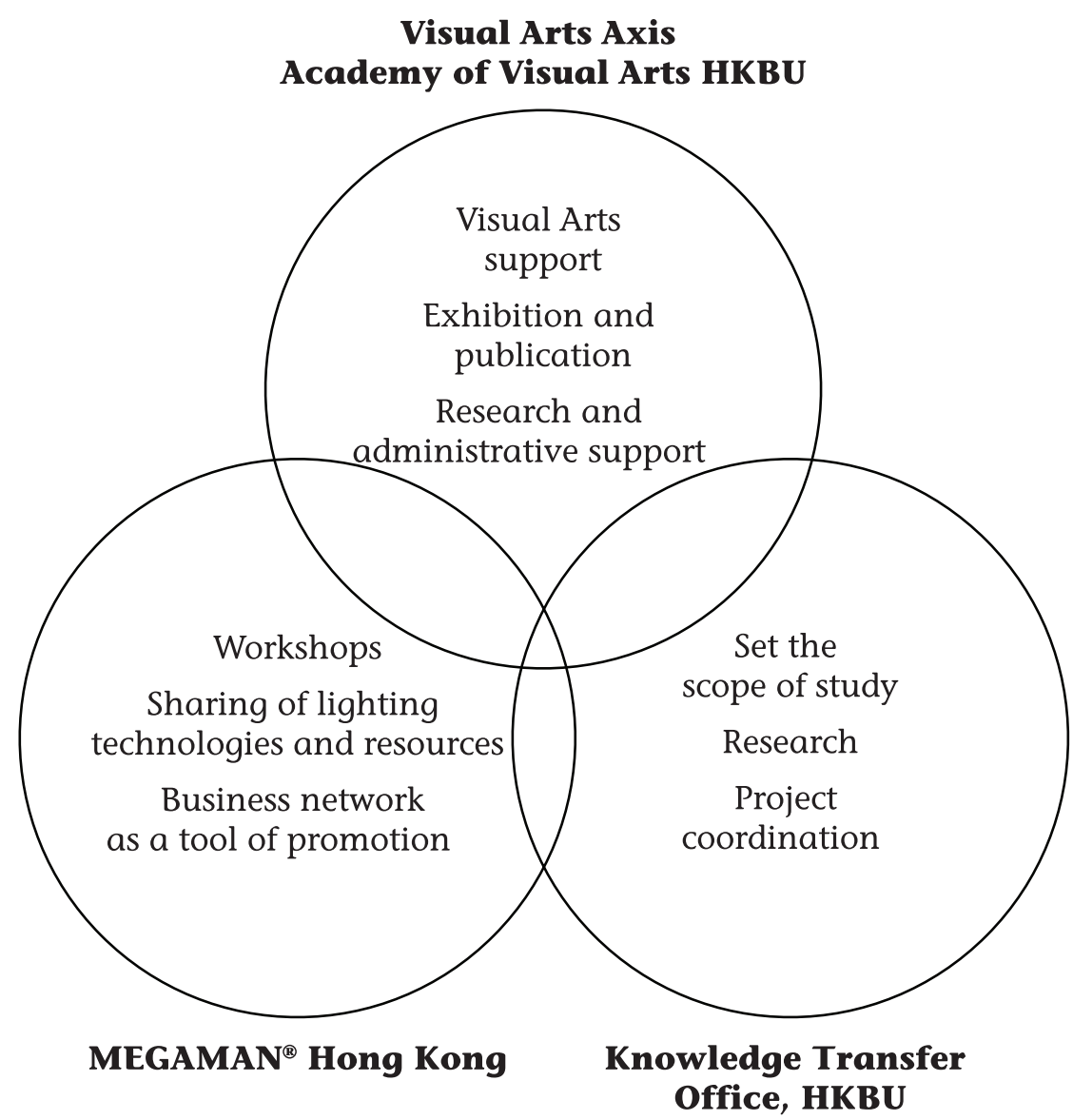

The 'In Light of Visual Arts' project was more than a simple transfer of technological knowledge of eco-lighting from MEGAMAN® Hong Kong to AVA students; it also aimed to make this knowledge available to a wider audience, for instance, by holding an exhibition and educating people about different applications of eco-lighting and the associated technology.

\section{KNOWLEDGE TRANSFER AND THE ARTS}

Smith (2012) points out that disciplines within academic institutions tend to have a specific or, in negative terms, 'narrow' field of study and that, through knowledge transfer, academics from different departments would be able to break through their established knowledge boundaries to enhance interdisciplinary collaboration, as well as communicating and cooperating with industry. As Ozga and Jones 2006 (p. 7) note, '... recent academic 
Figure 2: Clusters available for visual arts students explorations of the nature of knowledge have included discussion of the relationship between the kind of knowledge in play and its transfer and transferability'.

The 'In Light of Visual Arts' project aimed to examine how knowledge transfer could be integrated with Informal Learning approaches and visual arts elements, in addition to scientific and technical knowledge, which most people would associate with lighting. 'Indeed, [d] evelopments in understanding knowledge support a shift from traditional linear models of knowledge production and subsequent dissemination to an interactive, iterative, problem-focused, trans-disciplinary model.' (Ozga \& Jones 2006, p. 9). Knowledge transfer should not be limited to the knowledge related to technical development, it should also '... include knowledge that can promote critical political and social awareness and understanding' (Ozga \& Jones, 2006, p. 9). There seems on the surface to be little scope for knowledge transfer for the arts, humanities and social sciences disciplines, yet these disciplines may actually be able to contribute to the creation of meaning and value beyond economic outcomes. Thus, knowledge transfer from these disciplines could provide other perspectives for visual arts students, such as on environmental issues, which may not be included in their studies.

The participants in the 'In Light of Visual Arts' project were students of Bachelor of Arts (Hons) in Visual Arts, an undergraduate program offered by AVA. According to the program requirements, students must take some compulsory courses, which include Introduction to Visual Arts, Introduction to Western Art and Introduction to Chinese Art. Apart from the introductory courses, the students have to choose at least three clusters, each comprising four to five courses, from three divisions.

\begin{tabular}{lll}
\hline $\begin{array}{l}\text { Studio and Media } \\
\text { Art Division }\end{array}$ & $\begin{array}{l}\text { Visual Arts Studies } \\
\text { Division }\end{array}$ & $\begin{array}{l}\text { Craft and Design } \\
\text { Division }\end{array}$ \\
\hline 1. Drawing and & 1. Art History \& & 1. Graphic Art \\
Painting & Theory & 2. Experimental \\
2. Chinese Arts & 2. Visual \& Material & Imaging \\
3. Lens-based Media & Culture & 3. Glass \& Ceramics \\
4. Sculpture & & 4. Wearables \\
5. Body & & 5. Objects \& \\
& & Environment \\
\hline
\end{tabular}

Within the 12 available clusters, only 3 cover knowledge and use of lighting: Lens-based Media, Experimental Imaging, and Objects and Environment. The use of lighting has only a supportive role in the art media. For example, 'Looking Through the Lens', a course within the cluster Lens-based Media, covers lighting and optical theories and is an accessory to photography, investigating how light affects the photo-taking process. 'Sustainable Design', a course within the cluster Objects and Environment, covers sustainability in design by utilising recyclable or biodegradable materials. The course structure therefore inspired 
the writer to consider how lighting knowledge and eco-philosophy, or any other relevant factors, could be articulated in the BA (Hons) in Visual Arts program, so that lighting, as an important source of knowledge for visual arts students, could sharpen students' observation and sensitivity towards the community and environment around them, thus forming the basis for unique learning and artistic experiences. The provision of opportunities for unique experiences is important as internship may not be an integral component of all undergraduate programs. Where this is the case, this could be facilitated by working with different stakeholders within the commercial sector. For example, in the 'In Light of Visual Arts' project, student participants working with an established company of the lighting industry, MEGAMAN®, were guided to consider the needs of various business and governmental organisations: how a commercial organisation works towards an ethical and sustainable business strategy, and how that strategy may be linked to the arts, where the arts and sustainable business share a common belief in social and community betterment.

The KT process for 'In Light of Visual Arts' involved two key stages. The first stage was knowledge transfer from MEGAMAN® Hong Kong to student participants; the second stage was the transfer of knowledge from students to the general public - how AVA students and lecturers incorporated lighting knowledge so that in the long run it might become an intangible asset for both the general public and MEGAMAN® Hong Kong, the lighting manufacturer.

\section{KNOWLEDGE TRANSFER AND 'IN LIGHT OF VISUAL ARTS'}

\section{First Stage of Knowledge Transfer Process}

MEGAMAN® Hong Kong played an important role in the first stage of the knowledge transfer process of this project. Through three workshops and a concept store visit, the lighting company transferred knowledge about eco-friendly lighting equipment and solutions to AVA student participants. Students were thus able to combine their artistic knowledge and talent with the expertise gained from MEGAMAN ${ }^{\circledR}$ Hong Kong throughout their creative activities. They were expected to have some knowledge and understanding of lighting technology and of how the use of lighting maximises the technology's efficiency while minimising potential hazards to the environment.

Whereas it is generally perceived that visual arts students have a good eye and hand for creative processes, they are also expected to tackle aspects of these processes they are less familiar with, but that are important when it comes to art and ethics, or the more encompassing genre known as 'Environmental Art'. These are not out-of-reach experiences related to seeing art as mere cultural capital or classy commodity, but echo the third and fourth objectives of the project, to 'raise and gain awareness of HKBU's contribution to visual communication and the environment' and to 'stimulate knowledge transfer in the arts 


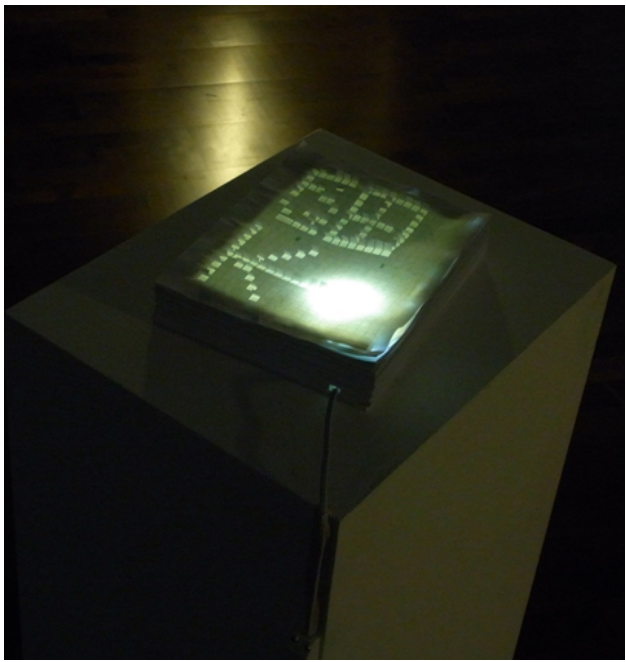

Figure 3: Dear teacher, 2012 and humanities through collaboration and partnerships'. It was therefore important for the visual arts students involved in the project to acquire lighting knowledge and to disseminate what they had learned in a comprehensible manner. This is not to say that the aim should not be art creation, but rather that the art should become more approachable for the general public so that community members are not restrained by 'technical boundaries'. This acts as a good beginning for disseminating both artistic and lighting knowledge, both being similar in the techniques they involve.

This explains how the project, in enabling an exploratory experience for its student participants, encouraged them to use lighting knowledge in dynamic ways for artistic creation while attaching meaning to it, both personal and social. For example, the making of light and shadow was a fruitful experiment for some student participants - by making full use of the low temperature characteristics of light-emitting diode (LED) products, such a process avoids the burn that may be caused to the thin layer of paper or wood of lighting products when put close to the surface.

The work, Dear teacher (Figure 3), for example, was made from a large pile of genko yoshi, a paper often used for Chinese writing. In putting the light bulb underneath the pile of papers, the student was required to consider the capability of the papers and the lighting product, as well as the heating issue. The student was also required to incorporate the skill of paper crafting with the interplay of light and shadow, working to articulate the piece on both personal and public levels - to communicate with the general public. Similar examples include The full moon, with its display of five decoupage lanterns, each decorated with patterned utensil napkins, and An interplay of light and characters, a work expressing its literal meaning. It is a white cloth installation with Chinese characters presented in a free-floating order while casting light on the cloth. The exploration of light and shadow demonstrates how lighting products mean more than illumination - articulating certain art forms, while being a stable and sustainable lighting source. The mechanism behind the art-making process also engages the general public, prompting student participants to design with various exhibition spaces in mind, as well as audiences.

\section{Informal Learning and knowledge transfer.}

As discussed above, MEGAMAN® Hong Kong covered practical knowledge about lighting technology and an introduction to lighting in its three workshops and provided an opportunity for students to gain on-site experience of different lighting functions, as well as knowledge about choice of products and skills. Knowledge transfer as effected in 'In Light of Visual Arts' thus differs from conventional knowledge transfer in that the emphasis is more on student application and how this can be used in the development of a feasible model for visual arts education in the longer term. 
Figure 4: Krathwohl's revised Bloom's Taxonomy
In light of this, the revised Bloom's Taxonomy of Educational Objectives, proposed by Anderson and Krathwohl (Krathwohl 2002), and which includes six major learning objectives (Figure 4), has been used as a framework for a visual arts educational model.

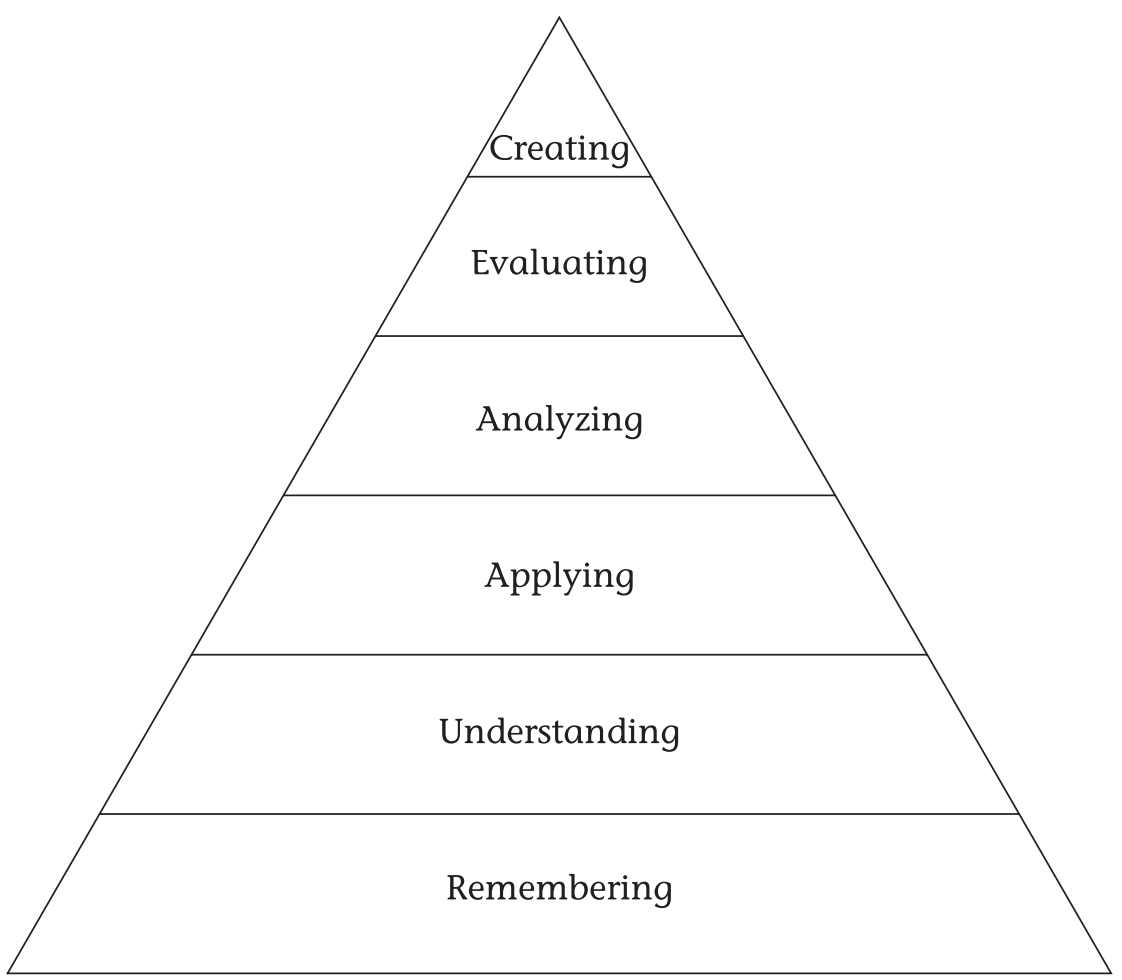

The content and activities in workshops 1 and 2 relate to the 'Remembering' and 'Understanding' categories in Figure 4, as the student participants were required to memorise and understand the information and concepts conveyed by the representatives of MEGAMAN® Hong Kong about their lighting products. Different cognitive levels of the Revised Bloom's Taxonomy played an important role during workshop 3 and the art-making process, where students had to give careful consideration to fusing knowledge of visual arts and lighting - thus reaching beyond creating merely 'glowing' artwork. Student participants were to apply the lighting knowledge they had acquired from the workshops and to analyse which, if not all, would fit their works better. The three works mentioned above all utilise paper and lighting sources, but how these mediums are individually integrated produces three different effects. Students have to evaluate how to maximise the impact of lighting before coming up with their own creation: in An interplay of light and character, by displaying words in free-floating order; and in The full moon by using tailored patterned napkins for five decoupage lanterns.

In addition to the integration and application of different concepts, the project aimed to consolidate the KT process with actual creative experiences. John Dewey, the renowned educator and philosopher, proposed theories emphasising the relationship between experiences and creativity. Dewey believed that knowledge should be obtained through different experiential or creative experiences, and that experiences were the interactions between people and the environment. 'Learning by doing', according 
to Dewey, was an important way of learning and ensured that students were able to make good use of their knowledge in daily experiences. Dewey (1916) suggested that students, also as learners, should be allowed to participate in various learning activities to acquire a variety of knowledge, which supports the aim of 'In Light of Visual Arts' to bring the visual arts and the use of lighting closer together. Sustainable lighting is a significant development in modern societies, although generally it appears to be detached from the art-making process and mostly seen as a separate professional domain.

Lacking the inclusion of lighting knowledge in visual arts education partly explains the difficulties participating students encountered in the course of its trial. Dewey (1934) pointed out that, when learners are required to inquire into a process they have gone through where they have encountered problems or obstacles to learning, reflection could lead them to come up with solutions, and the final solution itself would often signify the formation of new knowledge. When student participants in the project were required to assess if their artistic ideas were feasible, having made their own artworks with resources that may have been quite unfamiliar to them, they had to understand and differentiate between various lighting products (or theories) before refining their work. Lighting products became part of the art materials or essential elements of their artwork, although they may have overlooked or taken them for granted previously.

During the process of exploration, even though the students received advice and suggestions from different parties, such as representatives from MEGAMAN® Hong Kong or AVA lecturers, they sometimes still had questions or problems when engaging in the art-making process. In addition to the problems during the production process, mentioned previously, such as choice of lighting product colour, once preparing for the exhibition the students faced other kinds of challenges related to, for example, assembling their artworks in a limited exhibition space, which had an impact on how their original ideas could be viewed. Students then had to make adjustments or amendments to their artworks, while retaining the original concepts as much as possible.

Davies (2008) further investigated experiential learning and came to believe that it could be categorised as a form of Informal Learning (Figure 5). He pointed out that this kind of learning was affected by a number of elements, apart from observation and conceptualisation, including formal knowledge, past experiences and expectations.

Hayton, Boyes and Preston (2010) point out that creative knowledge transfer should not be limited to others who are involved in the creative process. Creative knowledge transfer is an iterative process and provides lots of opportunities for all learners to reflect on the experience and knowledge gained during the process. 
Figure 5: Overview of the elements involved in Informal Learning

\author{
Expectations \\ Emotions \\ Opportunity \\ Learning orientation, \\ including memory \\ Own observations \\ Fellow participants' \\ observations \\ Informed non-participants' \\ observations \\ Formal knowledge \\ Own experience \\ Reflection \\ Insight \\ Credibility checking \\ Experience bank \\ Experience
}

'In Light of Visual Arts' adopted the Informal Learning approach. Not only did students 'receive' the lighting knowledge being transferred to them from MEGAMAN® Hong Kong, they were also able to construct new knowledge by taking part in the project. The learning experience was enhanced by acquiring new knowledge, incorporating the new knowledge into the art-making process and subsequently disseminating the artworks to a wider audience, both art lovers and the general public.

\section{Feedback from student participants.}

Student participants revealed that they got a taste of what it was like applying visual arts in the commercial world. For example, one of the students came up with an original idea for creating a light with adjustable brightness; however, the participant was compelled to compromise his creative ideal due to his limited technical knowledge. As a result, he modified his product to be a conventional lamp with an ordinary switch. This example illustrates the imperative role technical support plays in helping students solve problems encountered in the creative process. Due to such problems with implementation, some students have suggested incorporating workshops on basic electrics in forthcoming KT projects, especially those that relate closely to art and design. Other students suggested inviting professional artists who were familiar with light as a creative medium to be consultants.

These suggestions reinforce the importance of technical knowledge. Even a basic knowledge of lighting made it possible for participants in the project to enhance their creative process by enabling them to utilise their knowledge of light more flexibly, more thoroughly, and to greater success.

\section{Second Stage of Knowledge Transfer Process}

With the integration of the Informal Learning approach, the AVA students managed to consolidate the knowledge transferred from MEGAMAN® Hong Kong to create 11 artworks incorporating 
visual arts and lighting elements. Stichler (2011, p. 19) remarks that, for any KT project between different disciplines, it is important to have 'dissemination of "the good, the bad, and the ugly", what worked well and what did not work to our expectations, and both supported and failed hypotheses convey knowledge of these findings to others'.

Participants in the 'In Light of Visual Arts' project agree with this notion, believing that knowledge transfer should not be confined to commercial industry or academia, and that the project content, process and outcomes should be shared with the general public so as to become an extension of the knowledge transfer partnership. The University Grants Committee of Hong Kong SAR (2013) also agreed that knowledge transfer should be a reciprocal process: the community should benefit by gaining knowledge on lighting, while the collaboration between university and industry should draw the two parties closer together.

The aim of Visual Arts Axis, an organisation affiliated with AVA, mentioned earlier, was to reach out to the community by means of visual arts. Visual Arts Axis therefore invited a number of local secondary schools to the 'In Light of Visual Arts' exhibition, and provided guided tours for them. By this means, secondary school students had the opportunity to learn more about the basics of lighting, mainly the difference between eco-friendly lighting products and traditional ones, as well as its impact on artmaking and, in turn, the environment. However, arranging visits and guided tours of the exhibition for secondary school students was a fairly obvious source of community outreach for this project. Knowledge transfer could have been enhanced by introducing more proactive measures to engage other stakeholders within the community at an earlier stage of the project.

\section{CONCLUSION}

As a pilot project aimed at synthesising lighting knowledge, visual arts and community engagement, 'In Light of Visual Arts' was ambitious in steering away from a unidirectional approach, which is often the favoured model for 'technology transfer'. The project nonetheless prompted student participants and the writer to attempt to enhance the function of the visual arts, at the same time bringing visual arts students, artists, art-lovers and, if possible, the general public closer, using the 'In Light of Visual Arts' project as the medium.

While lighting knowledge is (mostly) technical, and thus functional, the information provided by the lighting manufacturer also catered to the creative needs of visual arts students: how to comprehend the concept of illumination, temperature and space. While some artists may be offended by the notion of functionality in art, this project indicates that both function and creativity can play a part in bringing environmental messages to a broader audience through a knowledge transfer partnership. Including lighting in the visual arts curriculum and/or as an extracurricular 
activity, in line with the Informal Learning approach, could inspire future visual arts students and other participants in the project to come up with other approaches as to how the combination of lighting and visual arts knowledge could have a stronger impact in enhancing community awareness of the environment.

\section{REFERENCES}

Davies, L 2008, Informal learning: A new model for making sense of experience, Gower, Aldershot, UK.

Dewey, J 1916, Democracy and education, The Free Press, New York.

Dewey, J 1934, Art as experience, Perigee Books, New York.

Hayton, S, Boyes, L \& Preston, Y 2010, Creative labs: Exploring creative knowledge transfer, Hayton Associates, Bradford, UK.

Knowledge Transfer Office 2013, 'What is knowledge transfer', Knowledge Transfer Office, Hong Kong Baptist University, viewed 3 July 2013, http:// kto.hkbu.edu.hk/eng/channel.php?channel=what-is-knowledge-transfer.

Krathwohl , D 2002, 'A revision of Bloom's Taxonomy: An overview', Theory Into Practice, vol. 41, no. 4, pp. 212-18.

Ozga, J \& Jones, R 2006, 'Travelling and embedded policy: The case of knowledge transfer', Journal of Education Policy, vol. 21, no. 1, pp. 1-17.

Smith, L 2012, Knowledge transfer in higher education: Collaboration in the arts and humanities, Palgrave Macmillan, Hampshire, UK.

Stichler, J 2011, 'Knowledge transfer through dissemination', HERD, vol. 4, no. 2, pp. 17-22.

UGC (University Grants Committee) 2012, Knowledge transfer, viewed 28 December 2012, www.ugc.hk/eng/ugc/activity/kt/kt.htm. 